\title{
Bilateral recurrent external obturator muscle hematoma: An unusual cause of pelvic pain in hemophilia
}

\author{
TANER ARPACI ${ }^{1}$, ILGEN SASMAZ $^{2}$, TUGANA AKBAS $^{1}$, ALPER EKEN $^{3}$, ANIL OZGUR $^{4}$ and BULENT ANTMEN ${ }^{5}$ \\ ${ }^{1}$ Department of Radiology, Acibadem Adana Hospital, Acibadem University, 01130 Adana; ${ }^{2}$ Department \\ of Pediatric Haematology, Cukurova University, 01330 Adana; ${ }^{3}$ Department of Urology, Acibadem Adana Hospital, \\ Acibadem University, 01130 Adana; ${ }^{4}$ Department of Radiology, Mersin University, 33110 Mersin; \\ ${ }^{5}$ Department of Pediatric Haematology, Acibadem Adana Hospital, 01130 Adana, Turkey
}

Received September 22, 2015; Accepted January 8, 2016

DOI: $10.3892 / \operatorname{mco} .2016 .736$

\begin{abstract}
Following joint hemorrhages, intramuscular hemorrhages are the second most prevalent bleeding pattern in hemophiliac patients. Hematomas of the iliopsoas muscle are a well-known complication of hemophilia; however, obturator muscle hematomas are rare. We herein report a case of spontaneous bleeding of the bilateral external obturator muscles, which occured three times within a period of 9 months in a hemophilia patient with factor VIII inhibitors. To the best of our knowledge, this is the first published case of an obturator externus muscle hematoma in hemophilia. In addition to hip hemarthrosis, iliopsoas hematomas and acute appendicitis, obturator muscle hematoma should be considered as one of the diagnostic alternatives for pelvic pain in hemophilia $\psi$ patients. Magnetic resonance imaging enables rapid diagnosis of obturator muscle hematoma.
\end{abstract}

\section{Introduction}

Intramuscular hematomas represent $10-25 \%$ of all hemorrhagic complications in patients with hemophilia (1). Factor VIII (FVIII) replacement therapy is crucial to the therapeutic and conservative treatment of hemorrhagic complications. However, the emergence of FVIII inhibitors is a major complication in haemophilia. Upon the occurrence of a hemorrhagic event, subsequent events may become unpredictable and their management becomes more demanding in hemophiliac patients with factor VIII inhibitors.

The diagnosis of pelvic pain may also be challenging in patients with hemophilia. Under such conditions, evaluation should be based on three main pathological findings: hip hemarthrosis, iliopsoas hematoma and acute digestive tract

Correspondence to: Dr Taner Arpaci, Department of Radiology, Acibadem Adana Hospital, Acibadem University, 66 Cumhuriyet Caddesi, Seyhan, 01130 Adana, Turkey

E-mail: tanerarpaci@yahoo.com

Key words: haemophilia, pelvic pain, obturator externus muscle, intramuscular hematoma, magnetic resonance imaging illness, such as appendicitis or diverticulitis. However, rare diagnoses must be considered. Obturator muscle hematomas are very rarely encountered. Aouba et al reported two cases of spontaneous obturator internus muscle hematoma in hemophilia, which is the only publication regarding obturator muscle hematomas in the literature (2). To the best of our knowledge, cases of obturator externus muscle hematoma in the context of hemophilia have not been published to date. We herein report a case of spontaneous bleeding of the bilateral obturator externus muscles, which occurred three times within a period of 9 months in a hemophilia patient with factor VIII inhibitors. The most frequently published cases involving the obturator muscles are primary pyomyositis or intramuscular abscess (3-6). We also underline the possibility of underdiagnosing obturator muscle hematoma with ultrasonography, which requires more advanced imaging modalities, such as magnetic resonance imaging (MRI) or computed tomography (CT).

\section{Case report}

A 22-year-old male patient diagnosed with haemophilia A (FVIII <1\%) with high responding inhibitors $(6.4 \mathrm{BU})$ for 2 years, was admitted to our emergency department with left hip, groin and thigh pain, extending to the pubic area. At rest, the patient maintained his hip in mild flexion, whereas movement resulted in pain and muscular spasm. The psoas test was within normal limits. The findings on examination of other joints, cardiovascular and respiratory systems were normal. Routine radiographs and ultrasonography of the pelvis and hips revealed no abnormalities. A pelvic MRI revealed extensive bleeding and edema in the left obturator externus muscle (Fig. 1A and B). The clinical findings subsided following initiation of treatment with recombinant activated FVII (rFVIIa). The treatment included $90 \mathrm{mcg} \mathrm{kg}^{-1}$ every $3 \mathrm{~h}$ for 3 days. The control MRI performed 3 weeks after the first hemorrhagic event revealed regression of the intramuscular hematoma and edema (Fig. 1C and D).

The patient was admitted to the emergency department with right hip, groin and thigh pain 5 months after the first event. The musculoskeletal findings were nearly identical to the first presentation, when the patient was admitted to the hospital. An MRI revealed a focal hematoma and mild peripheral edema in 

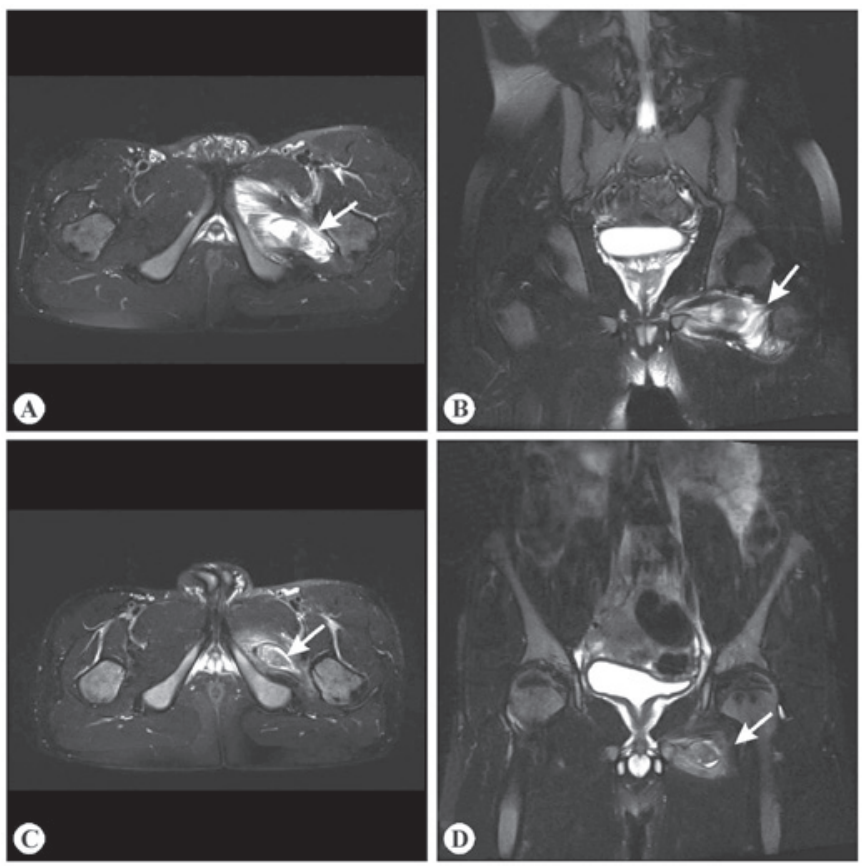

Figure 1. Fat-supressed T2-weighted (A) axial and (B) coronal magnetic resonance images of the pelvis, demonstrating a hematoma and peripheral edema in the left obturator externus muscle. Fat-supressed T2-weighted (C) axial and (D) coronal magnetic resonance images obtained 3 weeks after the first bleeding event revealed regression of the intamuscular hematoma and edema.

the right obturator externus muscle (Fig. 2A-B). The symptoms were relieved following treatment with rVIIa $\left(90 \mathrm{mcg} \mathrm{kg}^{-1}\right.$ every $3 \mathrm{~h}$ for 4 days).

The patient was again admitted to the emergency department with right iliopelvic pain and identical musculoskeletal findings 4 months after the second event. An MRI revealed a larger hematoma and prominent edema in the right obturator externus muscle (Fig. 2C and D). The clinical findings were alleviated following treatment with rFVIIa $\left(90 \mathrm{mcg} \mathrm{kg}^{-1}\right.$ every $3 \mathrm{~h}$ for 4 days).

The patient provided written informed consent for the publication of the details of this case.

\section{Discussion}

The majority of the hemorrhagic complications in hemophiliac patients occur within the musculoskeletal system. Following joint hemorrhages, intramuscular hemorrhages are the second most prevalent bleeding pattern in haemophilia. Hemorrhaging generally occurs following trauma, although it may also occur spontaneously (7). The clinical findings are based on the affected muscle, its dimensions and the extent of the coagulation defect. Bleeding causes a soft swelling in the muscle, quickly progressing into defensive spasm and leading to flexion of the adjacent joint, with limitation of movement and intense pain. A limited number of studies have been conducted on the pathology of the mentioned muscle hematomas. It is considered that muscle necrosis happens quickly due to ischemia of the fibres as the developing hematoma damages the neurovascular bundles (8).

A significant location of hematoma occurrence is the iliopsoas muscle; this is a well-known complication of hemophilia
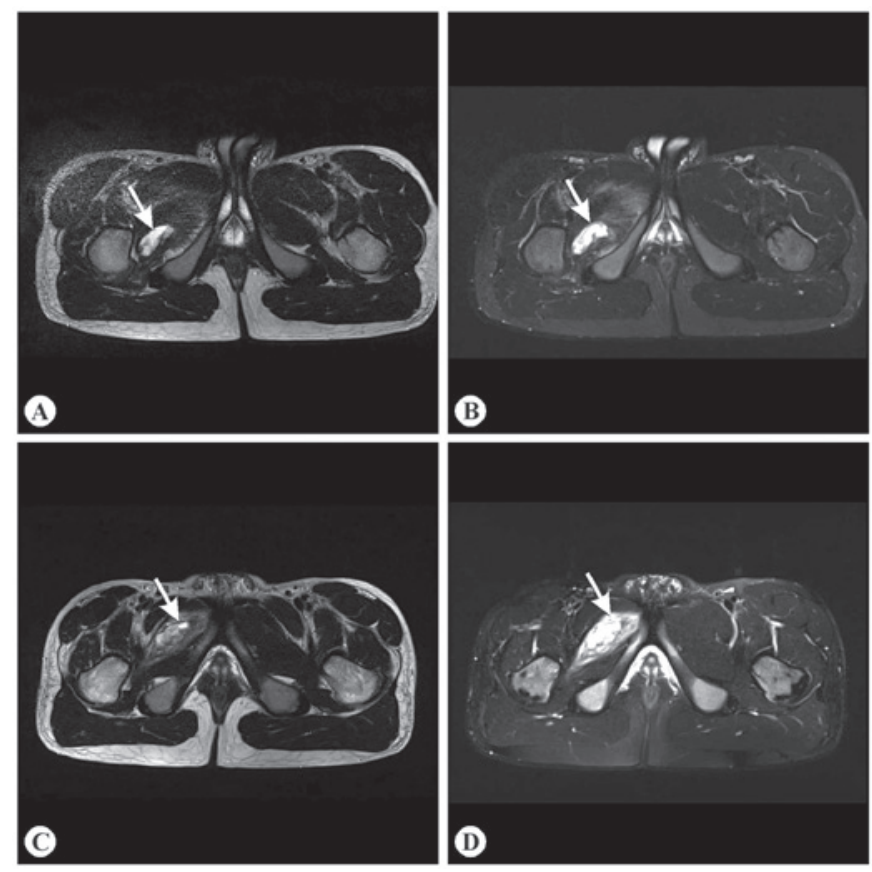

Figure 2. (A) T2-weighted axial and (B) fat-supressed T2-weighted axial magnetic resonance (MR) images obtained 5 months after the first bleeding event revealed a focal hematoma and mild peripheral edema in the right obturator externus muscle. (C) T2-weighted axial and (D) fat-supressed T2-weighted axial MR images obtained 4 months after the second bleeding event demonstrated a larger hematoma and prominent peripheral edema in the same muscle.

and is considered as a possibly life-threatening complication, significantly associated with morbidity. Iliopsoas haematoma leads to compression of the femoral nerve due to the developing lesion in the muscle; there is generally no traumatic etiology and the first occurrence is sudden, with intense lower abdominal or groin pain (9).

The obturators are the pelvi-trochanteric muscles originating from the posterior (obturator internus) and the anterior (obtutrator externus) walls of the obturator foramen. These two muscles attach to the greater trochanter of the femur and they are involved in thigh external rotation and abduction, or external rotation and adduction respectively. The differential diagnosis of the obturator externus muscle hematoma should also include primary pyomyositis or abscess. However, only two cases of isolated obturator externus muscle abscess have been reported in the literature to date (5-6). In intramuscular abscesses, fever and elevation of the erythrocyte sedimentation rate or C-reactive protein levels are likely to occur. An MRI with intravenous gadolinium administration achieves a rapid diagnosis of muscle inflammation and rim-enhancing abscess formation; it also enables the detection of additional local pathologies (5).

Ultrasonography is generally of little value in the diagnosis of obturator muscle hematomas, contrary to pelvic MRI, as mentioned in the present case. The CT findings of the intramuscular hematoma are non-spesific asymmetric muscular hypertrophy and possible blood attenuation in the muscle (2). MRI demonstrates hemorrhagic signal abnormality and edema in the affected muscle (10).

As a result, in addition to hip hemarthrosis, iliopsoas hematomas and acute appendicitis, obturator muscle hematoma 
must be considered as one of the diagnostic alternatives for pelvic pain in patients with hemophilia.

\section{References}

1. Beyer R, Ingerslev J and Sørensen B: Current practice in the management of muscle haematomas in patients with severe haemophilia. Haemophilia 16: 926-931, 2010.

2. Aouba A,Breton S, Harroche A, Sy-Bah D, Torchet MF, Frenzel L, Lasne D, Padovani JP, Odent T and Rothschild C: Spontaneous obturator internus muscle haematoma: A new unpublished cause of iliopelvic pain in haemophilia. Haemophilia 19: 157-160, 2013.

3. Nikolopoulos DD, Apostolopoulos A, Polyzois I, Liarokapis S and Michos I: Obturator internus pyomyositis in a young adult: A case report and review of the literature. Cases J 2: 8588, 2009.

4. King RJ, Laugharne D, Kerslake RW and Holdsworth BJ: Primary obturator pyomyositis: A diagnostic challenge. J Bone Joint Surg Br 85: 895-898, 2003

5. Kumar A and Anderson D: Primary obturator externus pyomyositis in a child presenting as hip pain: A case report. Pediatr Emerg Care 24: 97-98, 2008

6. Fowler T and Strote J: Isolated obturator externus muscle abscess presenting as hip pain. J Emerg Med 30: 137-139, 2006.
7. Duthie RB: Muscle bleeds and consequences. In: The Management of Musculoskeletal Problems in the Hemophilias. Duthie R, Rizza C, Giangrande P and Dodd C (eds.). 2nd edition. Oxford University Press, Oxford, pp104-pp117, 1994.

8. Forbes CD: Clinical aspects of the genetic disorders of coagulation. In: Disorders of Hemostasis. Ratnoff OD and Forbes CD (eds). 3rd edition. WB Saunders Company, Philadelphia, pp138-pp185, 1996.

9. Fernandez-Palazzi F, Hernandez SR, DeBosch NB and De Saez AR: Hematomas within the iliopsoas muscles in hemophilic patients: The Latin American experience. Clin Orthop Relat Res: 19-24, 1996.

10. Yuh WT, Schreiber AE, Montgomery WJ and Ehara S: Magnetic resonance imaging of pyomyositis. Skeletal Radiol 17: 190-193, 1988. 